\title{
Enhancement of DFT-calculations at Petascale: Nuclear Magnetic Resonance, Hybrid Density Functional Theory and Car-Parrinello calculations
}

\author{
Nicola Varini ${ }^{\mathrm{a}, \mathrm{b}, \mathrm{c}, 1, *}$, Davide Ceresoli ${ }^{\mathrm{d}, 2}$, Layla Martin-Samos ${ }^{\mathrm{h}, \mathrm{e}}$, Ivan Girotto ${ }^{\mathrm{a}, \mathrm{f}}$, Carlo Cavazzoni $^{\mathrm{g}}$ \\ ${ }^{a}$ ICHEC, 7th Floor Tower Building Trinity Technology E Enterprise Campus, Grand Canal Quay, Dublin 2, Ireland \\ ${ }^{b}$ iVEC, 26 Dick Perry Ave, Kensington WA 6151, Australia \\ ${ }^{c}$ Research and Development, Curtin University, GPO Box U 1987, Perth, WA 6845, Australia \\ ${ }^{d}$ CNR Istituto di Scienze e Tecnologie Molecolari (CNR-ISTM), clo Dept. of Chemistry, University of Milan, via Golgi 19, 20133 Milan, Italy \\ ${ }^{e}$ CNR Istituto Officina Molecolare (CNR-IOM), c/o SISSA, via Bonomea 235, 34136 Trieste, Italy \\ ${ }^{f}$ International Centre for Theoretical Physics (ICTP), Strada Costiera 11, 34014 Trieste, Italy \\ ${ }^{g}$ CINECA, via Magnanelli 6/3, 40033 Casalecchio di Reno, Italy \\ ${ }^{h}$ University of Nova Gorica, Materials Research Laboratory, vipavska cesta 11C, 5270 Ajdovscina, Slovenia
}

\section{Abstract}

One of the most promising techniques used for studying the electronic properties of materials is based on Density Functional Theory (DFT) approach and its extensions. DFT has been widely applied in traditional solid state physics problems where periodicity and symmetry play a crucial role in reducing the computational workload. With growing compute power capability and the development of improved DFT methods, the range of potential applications is now including other scientific areas such as Chemistry and Biology. However, cross disciplinary combinations of traditional Solid-State Physics, Chemistry and Biology drastically improve the system complexity while reducing the degree of periodicity and symmetry. Large simulation cells containing of hun'dreds or even thousands of atoms are needed to model these kind of physical systems. The treatment of those systems still remains a computational challenge even with modern supercomputers. In this paper we describe our work to improve the scalability of 'Quantum ESPRESSO [1] for treating very large cells and huge numbers of electrons. To this end we have introduced an extra level of parallelism, over electronic bands, in three kernels for solving computationally expensive problems: the Sternheimer equation 'solver (Nuclear Magnetic Resonance, package QE-GIPAW), the Fock operator builder (electronic ground-state, package PWscf) and most of the Car-Parrinello routines (Car-Parrinello dynamics, package CP). Final benchmarks show our success in computing 'the Nuclear Magnetic Response (NMR) chemical shift of a large biological assembly, the electronic structure of defected amorphous silica with hybrid exchange-correlation functionals and the equilibrium atomic structure of height Porphyrins anchored to a Carbon Nanotube, on many thousands of CPU cores.

'Keywords: Parallelization, k-Points, Plane Waves DFT, Car-Parrinello, NMR, Exact exchange

\section{Introduction}

Understanding the details of atomic/molecular structures as 'well as dynamics of solid materials and biological systems, is 'one of the major challenges confronting physical and chemi'cal science in the early 21 st Century. Such knowledge has direct impact on important issues in our society like: the design, 'synthesis and processing of new either eco-friendly or high'efficiency materials; development of new energy sources; control of materials degradation and recycling; design of drugs for 'specific pathology.

The complexity of these areas of research is growing rapidly along with the need for computational tools to deal with such complexity. Computer simulation is the only way to study these large physical systems. However, extremely expensive largescale computational resources do not come for free. In addi-

\footnotetext{
*Corresponding author.

${ }^{1}$ E-mail: nicola@ivec.org

${ }^{2}$ E-mail: davide.ceresoli@istm.cnr.it
}

tion to the scientific value of the computer simulation, scientific codes must show good scalability and efficiency for access to world-class supercomputing facilities.

In this respect, parameter-free first principles (aka ab-initio) atomistic calculations in the framework of Density Functional Theory (DFT) [2, 3] are quite popular models, as they combine reasonable chemical accuracy with affordable complexity and good scalability. The current available codes implementing those models [1, 4, 5, 6, 7, 8] have enabled computer simulations at the frontier of science.

Computer technology is rapidly changing. The trajectory of major computer manufactures of the last few years illustrate that vendors are rapidly increasing the number of cores to build supercomputing technology, reducing the clock frequency of each single compute element. The most powerful supercomputers in the world are today equipped with million of cores capable of scheduling billions of threads concurrently. Without substantial updating, many legacy codes will not be able to exploit this massive parallelism. Nevertheless, such horsepower gives the opportunity to address problems of unprecedented 
size and complexity. This paper presents a parallelization strategy, based on the distribution of electronic band loops, for efficiently scaling calculations of hybrid-functionals and Nuclear Magnetic Resonance (NMR). In addition we describe how the same parallelization strategy impacts in the scalability of the Car-Parrinello computational kernels.

The paper is organized as follows. In Sec.2 2 we briefly review related work reported by other groups. In Sec. 3 we describe the equations implemented in the QE-GIPAW code to calculate NMR shielding tensors and in Sec. 4 the formulas needed to evaluate the Fock-exchange operator and energy. In Sec. 5 we outline the parallelization strategy based on bands and $q$-point distribution. In Sec. 6 we report and discuss benchmark results of our parallelization. Finally, in the last section we present our conclusions and perspectives on this parallelization strategy.

\section{Related work}

The idea of distributing computation over electronic bands was pioneered in the '90s by the CASTEP [14] code and was found to be effective on early vector machines (i.e. Cray Y-MP). In the subsequent years, the improvement of collective communication along with the availability of efficient FFT libraries, enabled a good scaling of plane wave codes up to few hundreds of CPUs with a simple two-level parallelization (k-points and plane waves). Only since very recently, top-level HPC machines have a huge number of CPU cores $\left(10^{4}-10^{5}\right)$ with limited amount of memory. As the number of atoms in the system increase, the number of k-points can be reduced, deteriorating the scaling and parallel efficiency of the codes. At this stage, in order to exploit the large number of CPU cores, an extra level of parallelism, electronic bands, must be introduced. The iterative diagonalization has been the first kernel to benefit of this strategy allowing a scalability up to few thousands of cores for electronic groundstate calculations within traditional exchangecorrelation functionals. ABINIT implemented a block preconditioned conjugate gradient algorithm [12], while VASP avoids explicit orthogonalization of bands by the RM-DIIS algorithm. Moreover, the QBOX [8] code was designed purposely for BlueGene machines by a clever data distribution and fully distributed linear algebra (ScaLAPACK [13]). Similar schemes have been implemented in CPMD, BigDFT, CASTEP [14] and are being implemented within different packages of the Quantum ESPRESSO distribution. Regarding the Exact Exchange (EXX) kernel, the NWCHEM code introduced an extra level of parallelism over occupied states and reported excellent scaling up to 2,048 CPUs [15]. In this work we report scaling results up to many thousands of CPU core, thanks to the band parallelization on replicated data of two of the most computationally intensive kernels (linear response and the Fock operator). In addition to this, we show that every step of the Car-Parrinello algorithm can be fully distributed (both data and computation), enabling petascale computation. This parallelization stragtegy allows to fully exploit the new EU Tier-0 petascale facilities such as CURIE [9], JUGENE [10] and FERMI [11].

\section{The GIPAW equations for the induced current and NMR shieldings}

The GIPAW method (Gauge Including Projector Augmented Wave) makes it possible to calculate the current induced by an infinitesimal external field, hence the NMR shielding tensors, in a periodic system by means of linear response. The GIPAW method is an extension of the PAW method [16] for reconstructing all-electron wavefunction and expectation values from a pseudopotential calculation. This is essential to compute accurately the response of the valence electrons in regions near the nuclei, which determines the NMR shielding.

The GIPAW method was formulated by Mauri [17] and Pickard [18, 19, 20] and we refer the reader to the original papers for the detailed derivation of the method. In the following, we show only the resulting set of equations for the induced current and outline the flow of the code.

We will start by noting that the GIPAW transformation of a quantum mechanical operator $O$ (eq. 17 of Ref. [18]), gives rise to two kind of terms. The first is the operator $O$ itself, (which we call bare term), acting on the valence wavefunctions all over the space. The second is a non-local operator acting only inside spherical augmentation regions centered around each atom. The non-local projectors enjoy the property of vanishing beyond a cutoff radius, equal to the cutoff radius of the pseudopotential. This term is called reconstruction term and is not computationally expensive, except for systems with more than 2000 atoms. For larger systems, work is in progress to block-distribute the non-local projectors and use the ScaLAPACK [13] library to perform linear algebra operations.

Therefore, in the following we will focus on the evaluation of the bare contribution to the induced current and to the magnetic susceptibility of the system. The induced current is defined as

$$
\begin{aligned}
\mathbf{j}_{\text {bare }}^{(1)}\left(\mathbf{r}^{\prime}, q\right)= & \frac{1}{c} \sum_{k} w_{k} \sum_{\mathbf{q}= \pm q \hat{x}, \pm q \hat{y}, \pm q \hat{z}} \frac{1}{2 q} \sum_{n \in \mathrm{occ}} \operatorname{Re}\left[\frac{2}{i}\right. \\
& \left.\left\langle u_{n \mathbf{k}}\left|\mathbf{J}_{\mathbf{k}, \mathbf{k}+\mathbf{q}}^{\mathrm{para}}\left(\mathbf{r}^{\prime}\right) \mathcal{G}_{\mathbf{k}+\mathbf{q}}\left(\epsilon_{n \mathbf{k}}\right) \mathbf{B} \times \hat{\mathbf{q}} \cdot \mathbf{v}_{\mathbf{k}+\mathbf{q}, \mathbf{k}}\right| u_{n \mathbf{k}}\right\rangle\right]
\end{aligned}
$$

where the first sum is over k-points with weight $w_{k}$. The second sum is over a star of q-points, where $q \ll 1$ is the inverse wavelength of the external magnetic field and $\hat{\mathbf{q}}$ is a unit vector. The third sum is over the occupied bands, $u_{n k}$ is the valence eigenstate at k-point $k$ and band index $n$. The bracket is evaluated from right to left, by applying first the non-local velocity operator

$$
\mathbf{v}_{\mathbf{k}, \mathbf{k}^{\prime}}=-i \nabla+\mathbf{k}^{\prime}+\frac{1}{i}\left[\mathbf{r}, V_{\mathbf{k}, \mathbf{k}^{\prime}}^{\mathrm{nl}}\right]
$$

to $\left|u_{n k}\right\rangle$, then by applying the Green's function, formally defined as

$$
\mathcal{G}_{\mathbf{k}+\mathbf{q}}(\epsilon)=\left(\mathcal{H}_{\mathbf{k}+\mathbf{q}, \mathbf{q}}-\epsilon\right)^{-1}
$$

where $\mathcal{H}_{\mathbf{k}^{\prime}, \mathbf{k}}=e^{-i \mathbf{k}^{\prime} \cdot \mathbf{r}} \mathcal{H} e^{i \mathbf{k} \cdot \mathbf{r}}$ is the periodic Kohn-Sham Hamiltonian. Then, we apply the paramagnetic current operator to the left to $\left\langle u_{n k}\right|$

$$
\mathbf{J}_{\mathbf{k}, \mathbf{k}^{\prime}}^{\text {para }}=-\frac{1}{2}\left[(-i \nabla+\mathbf{k})\left|\mathbf{r}^{\prime}\right\rangle\left\langle\mathbf{r}^{\prime}|+| \mathbf{r}^{\prime}\right\rangle\left\langle\mathbf{r}^{\prime}\right|\left(-i \nabla+\mathbf{k}^{\prime}\right)\right]
$$


Finally the braket product is evaluated and summed to obtain the current field. The induced magnetic field is obtained easily by the Biot-Savart law, after Fourier-transforming to reciprocal space:

$$
\mathbf{B}_{\text {bare }}^{(1)}=\frac{4 \pi}{c} \frac{i \mathbf{G} \times \mathbf{j}_{\text {bare }}^{(1)}(\mathbf{G})}{G^{2}}
$$

This procedure is repeated with $\mathbf{B}$ along each of the three Cartesian directions, and the bare NMR shielding tensor is obtained by evaluating the induced magnetic field response at each nuclear coordinate. The $\mathbf{G}=0$ term in eq. 5 depends on the macroscopic shape of the sample and the magnetic susceptibility tensor $\chi_{\text {bare }}$. The expression of $\chi_{\text {bare }}$ is very similar to eq. 1 .

$$
\begin{aligned}
\overleftrightarrow{Q}(q)= & -\frac{1}{c^{2}} \sum_{k} w_{k} \frac{1}{\Omega} \sum_{\mathbf{q}=q \hat{x}, q \hat{y}, q \hat{z}} \sum_{n \in \mathrm{occ}} \operatorname{Re}\left[\frac{1}{i}\right. \\
& \left.\left\langle u_{n \mathbf{k}}\left|\hat{\mathbf{q}} \times(-i \nabla+\mathbf{k}) \mathcal{G}_{\mathbf{k}+\mathbf{q}}\left(\epsilon_{n \mathbf{k}}\right) \mathbf{B} \times \hat{\mathbf{q}} \cdot \mathbf{v}_{\mathbf{k}+\mathbf{q}, \mathbf{k}}\right| u_{n \mathbf{k}}\right\rangle\right]
\end{aligned}
$$

where $\Omega$ is the cell volume and

$$
\begin{aligned}
& \overleftrightarrow{\chi}_{\text {bare }}=\frac{\overleftrightarrow{F}(q)-2 \overleftrightarrow{F}(0)+\overleftrightarrow{F}(-q)}{q^{2}} \\
& F_{i j}(q)=\left(2-\delta_{i j}\right) Q_{i j}(q) \quad i, j \in x, y, z
\end{aligned}
$$

Since the only difference between eqs. 1 and 6 is the operator to the left of the Green's function, the two formulas are evaluated back-to-back in the same loop.

Finally, the NMR shielding tensor $\overleftrightarrow{\sigma}(\mathbf{R})$ is obtained by adding the bare and reconstruction contributions of the induced magnetic field, evaluated at each nuclear position $\mathbf{R}$

$$
\overleftrightarrow{\sigma}(\mathbf{R})=-\left.\frac{\partial \mathbf{B}^{(1)}(\mathbf{r})}{\partial \mathbf{B}}\right|_{\mathbf{r}=\mathbf{R}}
$$

\subsection{The Sternheimer equation}

The most time-consuming operation in GIPAW is applying the Green's function (eq. 3) to a generic ket $\left|w_{n \mathbf{k}}\right\rangle$

$$
\left|g_{n \mathbf{k}+\mathbf{q}}\right\rangle=\mathcal{G}_{\mathbf{k}+\mathbf{q}}\left(\epsilon_{n \mathbf{k}}\right)\left|w_{n \mathbf{k}}\right\rangle
$$

To avoid inversion of large matrices or, equivalently, summation over all empty states, we solve instead the Sternheimer equation

$$
\left(\mathcal{H}_{\mathbf{k}+\mathbf{q}, \mathbf{k}}-\epsilon_{n \mathbf{k}}+\alpha P_{\mathbf{k}+\mathbf{q}, \mathbf{k}}\right)\left|g_{n \mathbf{k}+\mathbf{q}}\right\rangle=-Q_{\mathbf{k}+\mathbf{q}, \mathbf{k}}\left|w_{n \mathbf{k}}\right\rangle
$$

where $P_{\mathbf{k}+\mathbf{q}, \mathbf{k}}=\sum_{n \in \text { occ }}\left|u_{n \mathbf{k}+\mathbf{q}}\right\rangle\left\langle u_{n \mathbf{k}}\right|$ is the projector over the occupied manifold and $Q_{\mathbf{k}+\mathbf{q}, \mathbf{k}}=1-P_{\mathbf{k}+\mathbf{q}, \mathbf{k}}$ is the projector over the empty states. $\alpha$ is chosen as twice the bandwidth of the occupied states in order to make the $l h s$ positive definite.

Eq. 10 constitutes a set of $N$ independent linear equations, where $N$ is the number of occupied states and is solved for $\left|g_{n \mathbf{k}+\mathbf{q}}\right\rangle$ by the conjugate gradient (CG) method. In order to take advantage of level-3 BLAS operations, the CG update is performed initially on all electronic bands. Then, starting from the second iteration, the electronic bands are divided into two groups, each occupying a contiguous memory area: converged and not converged. A band is converged when the residual falls below a threshold $\left(10^{-7}\right.$ Rydberg). Therefore, we update only the bands which have not yet converged. Before returning from the subroutine, the bands are sorted according to the original band index $n$.

\subsection{Outline of the GIPAW code}

Before running an NMR calculation with the QE-GIPAW code [21], it is necessary to run a self-consistent calculation (SCF) with the PW code on a possibly relaxed structure, in order to obtain the ground state wavefunction $\left|u_{n \mathbf{k}}\right\rangle$. The QEGIPAW code reads from disk the wavefunctions and the electronic density generated by PW and computes the induced current and the magnetic susceptibility.

After an initialization phase, the QE-GIPAW code runs as follows:

\section{Loop over $k$-points}

1. Read $\left|u_{n \mathbf{k}}\right\rangle$ from disk, all bands

2. Set $q=0$ and for each $n$ and compute $\mathbf{v}_{\mathbf{k}, \mathbf{k}}\left|u_{n \mathbf{k}}\right\rangle$, $\mathcal{G}_{\mathbf{k}, \mathbf{k}}\left(\epsilon_{n \mathbf{k}}\right) \mathbf{v}_{\mathbf{k}, \mathbf{k}}\left|u_{n \mathbf{k}}\right\rangle$, and $(-i \nabla+\mathbf{k})\left|u_{n \mathbf{k}}\right\rangle$

3. Compute $Q(0)$ according to eq. 6

4. Loop over the star of $q$-points: $\mathbf{q}= \pm q \hat{x}, \pm q \hat{y}, \pm q \hat{z}$

1. Diagonalize the KS Hamiltonian at $\mathbf{k}+\mathbf{q}$

2. For each $n$ compute $\mathbf{v}_{\mathbf{k}+\mathbf{q}, \mathbf{k}}\left|u_{n \mathbf{k}}\right\rangle$, $\mathcal{G}_{\mathbf{k}+\mathbf{q}, \mathbf{k}}\left(\epsilon_{n \mathbf{k}}\right) \mathbf{v}_{\mathbf{k}+\mathbf{q}, \mathbf{k}}\left|u_{n \mathbf{k}}\right\rangle$, and $(-i \nabla+\mathbf{k}+\mathbf{q})\left|u_{n \mathbf{k}}\right\rangle$

3. Compute $Q(q)$ and $\mathbf{j}_{\text {bare }}^{(1)}\left(\mathbf{r}^{\prime}, q\right)$

2. Parallel execution only: reduce (parallel sum) $Q$ and $\mathbf{j}_{\text {bare }}^{(1)}$ over $k$-points and planewaves.

3. Solve the Biot-Savart equation (eq. 5), evaluate the induced magnetic field at each nuclear coordinates, output the NMR shielding tensors and terminate.

All reconstruction terms are evaluated after step 1.3 and step 1.4.3, with little computational cost. When employing ultrasoft [22] of PAW pseudopotential, there is an additional evaluation of the Green's function for each $k$ and $q$. The details can be found in Ref. [20].

Thus the flowchart of the code is based on three nested loops: over $k$-points, $q$-star and bands. Each term can be calculated independently and we anticipate that in order to run efficiently at the petaflop scale, we have distributed the calculation of every individual term on all the CPUs. Parallel communication is performed only at the end of the three loops and consumes little time. The only bottleneck is diagonalization step at $\mathbf{k}+\mathbf{q}$. We use the wavefunctions calculated at $\mathbf{k}$ as starting vector in order to reduce the number of Davidson or CG [1] iterations. Unfortunately the Davidson and CG algorithms cannot be parallelized easily over bands, because of the Grahm-Schmidt orthogonalization. We are currently seeking a diagonalization method which does not require and orthogonalization step, such as the RM-DIIS method [7].

Moreover, the explicit diagonalization can be avoided for very large simulation cells by choosing the magnitude of $q$ equal to the first reciprocal lattice vector, i.e. $q=2 \pi / a$, where $a$ is the lattice spacing of a cubic supercell. In fact, by the Bloch theorem, the wavefunctions at $\mathbf{k}+\mathbf{q}$ are given simply by:

$$
\left|u_{n, \mathbf{k}+\hat{\mathbf{q}} 2 \pi / a}\right\rangle=e^{i \hat{\mathbf{q}} 2 \pi / a \cdot \mathbf{r}}\left|u_{n \mathbf{k}}\right\rangle
$$


This method (which we call commensurate) provides an enormous speed-up and scaling for very large systems, but it may worsen the accuracy of the calculated NMR chemical shift. We are currently testing this method and results will be reported in a future paper.

\section{The Exact Exchange (EXX) within the planewave method}

After work of A. Becke in 1993, "A new mixing of HartreeFock and local density-functional theories" [23], it is nowadays becoming very common to include a fraction of Exact eXchange (formally Fock exchange) in Density Functional calculations. Exchange-correlation functionals with this fraction are called Hybrid-Functionals (HFs). The use of HFs enables at least a partial correction to the self-interaction error and the inclusion of a non-local contribution to the Hamiltonian. In other words, the inclusion of an EXX fraction, with respect to traditional DFT functionals, Local Density Approximation (LDA) or Generalized Gradient Approximation (GGA) [24], is mainly used to improve the agreement to experiments of the band gaps and the energetics of small molecules and solids.

The EXX energy contribution is defined as

$$
E_{x}=-\frac{1}{2} \sum_{\mathbf{k}} \sum_{\mathbf{q}} \sum_{i, j}^{o c c} \int d \mathbf{r} d \mathbf{r}^{\prime} \frac{u_{i \mathbf{k}}^{*}(r) u_{j \mathbf{k}-\mathbf{q}}(r) u_{j \mathbf{k}-\mathbf{q}}^{*}\left(r^{\prime}\right) u_{i \mathbf{k}}\left(r^{\prime}\right)}{\left|\mathbf{r}-\mathbf{r}^{\prime}\right|}
$$

which in reciprocal space reads

$$
E_{x}=-\frac{1}{2} \sum_{k} \sum_{i, j}^{o c c} \sum_{\mathbf{G}} \sum_{\mathbf{q}} \frac{M_{i \mathbf{k}, j \mathbf{k}-\mathbf{q}}^{*}(G) M_{i \mathbf{k}, j \mathbf{k}-\mathbf{q}}\left(\mathbf{G}^{\prime}\right)}{|\mathbf{G}+\mathbf{q}|^{2}}
$$

where $M_{i \mathbf{k}, j \mathbf{k}-\mathbf{q}}(\mathbf{G})=\mathrm{FT}\left[u_{j \mathbf{k}-\mathbf{q}}^{*}(r) u_{i \mathbf{k}}(r)\right]$ is the Fourier Transform of the products of two Bloch wavefunctions. These quantities are usually called "overlap charge densities". In planewave-based codes, the overlap charge densities are calculated via Fast Fourier Transform (FFT). From the reciprocal-space representation the wave-functions are Fourier transformed to real-space, the overlap product is calculated and then the result is transformed back to reciprocal space where the sums over occupied states, $\mathbf{q}, \mathbf{k}$, and $\mathbf{G}$ are performed [25, 26].

With the PWscf code of the Quantum ESPRESSO distribution, the convergence of the Khon-Sham (KS) Hamiltonian containing EXX fraction is achieved through two nested do loops. The inner loop converges the KS equations at fixed EXX potential while the outer updates the EXX potential $\left(V_{x}\right)$ and checks the overall accuracy. Before adding the first guess for $V_{x}$, PWscf makes a first full-self-consistent calculation with a traditional non-hybrid exchange-correlation functional. The EXX potential projected on an electronic state $\left|u_{i \mathbf{k}}\right\rangle$ is calculated as

$$
V_{x}\left|u_{i \mathbf{k}}\right\rangle=-\sum_{j}^{o c c} \sum_{\mathbf{G}} \sum_{\mathbf{q}} \frac{M_{i \mathbf{k}, j \mathbf{k}-\mathbf{q}}^{*}(G) M_{i \mathbf{k}, j \mathbf{k}-\mathbf{q}}\left(\mathbf{G}^{\prime}\right)}{|\mathbf{G}+\mathbf{q}|^{2}}
$$

It is easy to see that the distribution of the computational workload at the level of the sum over $j$ is very convenient as it just implies a reduction at the end of the loops.
Note however that in the current Quantum ESPRESSO implementation, hybrid functionals cannot be used to compute linear response quantities (i.e. NMR) because one should implement and solve simultaneously the coupled Hartree-Fock equations [27]. On the contrary, in pure DFT (no EXX) the zeroth order (Kohn-Sham) and first order (Sternheimer) equations are fully decoupled.

\section{Parallelization strategy}

Historically, the Quantum ESPRESSO distribution has been designed and used for modeling condensed-matter problems where the physical system can be mapped into a periodically repeated primitive cell. In these cases, the number of electrons (and consequently the number of electronic bands) is a relatively small number while the number of k-points (Brillouin zone sampling) is usually high. As a result, the k-points distribution across processes is the natural method of parallelization. Especially if considering that the DFT Hamiltonian is diagonal in $\mathrm{k}$ and the only source of communication arises from a sum in the calculation of the total energy. However, the boom of new organic and hybrid based electronics and optoelectronics materials (originated by the need of replacing silicon-based technologies) along with the fast evolution of supercomputers present brand-new scenarios extending the boundaries among condensed-matter, chemistry and biology. Most of the new topics involve systems with a huge number of atoms (subsequently, a huge number of electrons) and either low-symmetry (i.e., surfaces or wires) or no-symmetry at all (i.e., biological molecules).

The development activity focused on three main DFT applications: Nuclear Magnetic Resonance (NMR), Exact-Exchange (EXX) and Car-Parrinello calculations. Those computational models are implemented into the Quantum-ESPRESSO distribution among three scientific codes named QE-GIPAW [21], PWscf (EXact eXchange part only) and CP, respectively. For all these packages, performance analysis indicated that highly compute intensive sections were nested into loops over electronic bands. New levels of parallelism were introduced to better distribute such computation as these algorithms do not present data dependency along the band dimension. Applied, this simple concept becomes a breakthrough to simulate new scientific problems efficiently on large-scale supercomputers and to discover emerging physical effects that arise at a scale that was computationally unreachable before.

One of the most computationally intensive algorithms of the QE-GIPAW code is to evaluate the linear response of the wavefunctions to an external magnetic field. This is done by solving the Sternheimer equation which is composed of $n=1 \ldots N$ independent linear systems of the form

$$
\left(\mathcal{H}^{(0)}-E_{n}^{(0)}\right)\left|\Psi_{n}^{(1)}\right\rangle=\mathcal{H}^{(1)}\left|\Psi_{n}^{(0)}\right\rangle
$$

where $\mathcal{H}^{(0)}-E_{n}^{(0)}=\mathcal{G}\left(E_{n}\right)^{-1}$ is the inverse of the Green's function, $\mathcal{H}^{(1)}$ is the perturbing magnetic field, and $\left|\Psi_{n}^{(0)}\right\rangle$ are the unperturbed wave-functions, obtained by a previous SCF calculation with PWscf. Here $\left|\Psi_{n}^{(1)}\right\rangle$ are the unknowns and the index $n$ 


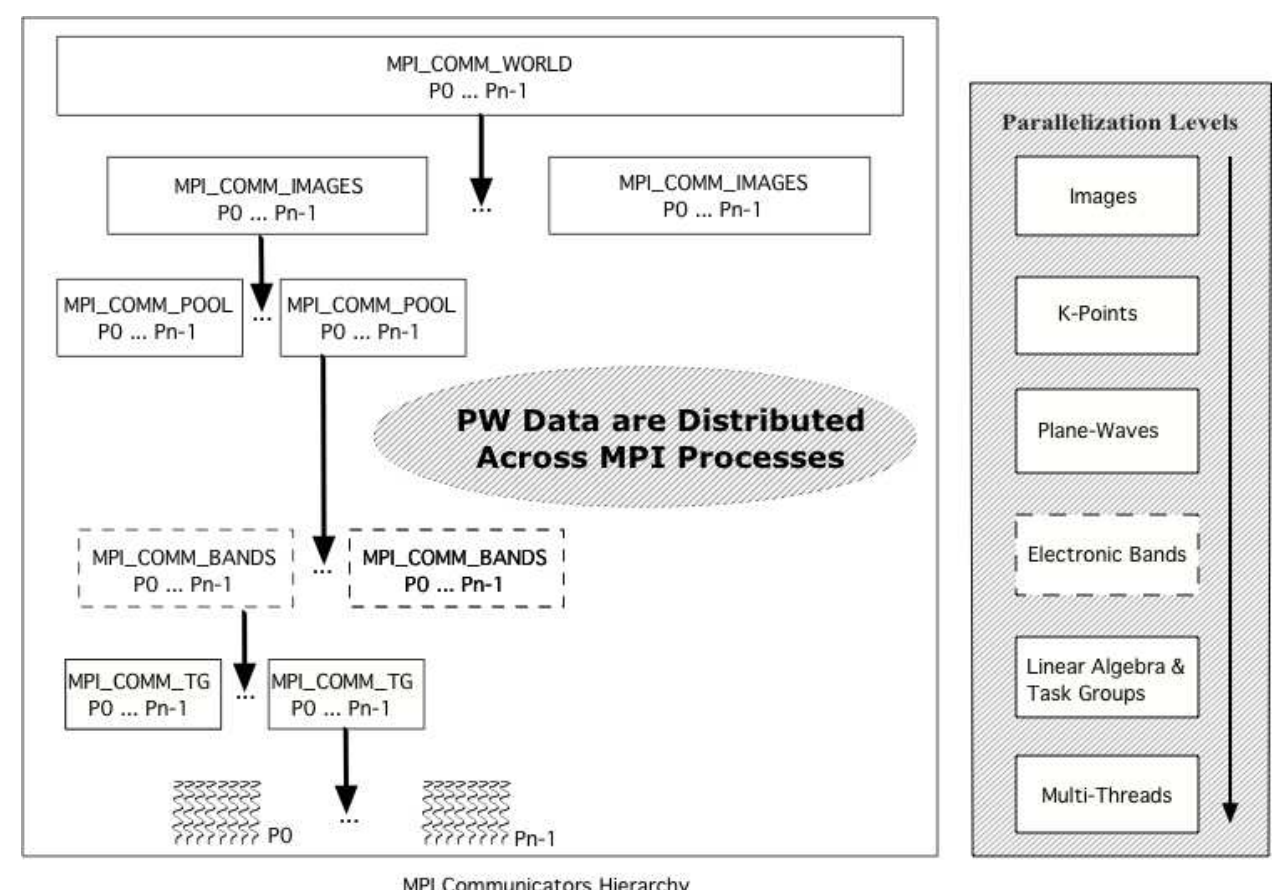

Figure 1: The picture stands for the levels of parallelism implemented within the Quantum ESPRESSO software packages. On the right hand side the hierarchy is logically represented from the higher to the deeper parallelism, expressed using multi-threading on top of MPI distribution. The left hand side shows how this hierarchy is mapped on MPI groups of processes (or communicators). From the top to the bottom four of these levels are divided in smaller groups from previous level (each black arrow stands for a splitting stage), starting from MPI COMM WORLD which represents all the MPI processes. For each sub-groups processes are identified from 0 to $\mathrm{n}-1$ where $\mathrm{n}$ is the number of processes for a given MPI communicator. At the last stage each process is no longer considered as a member of a set of processes (MPI communicator) but a single entity from which threads are created.

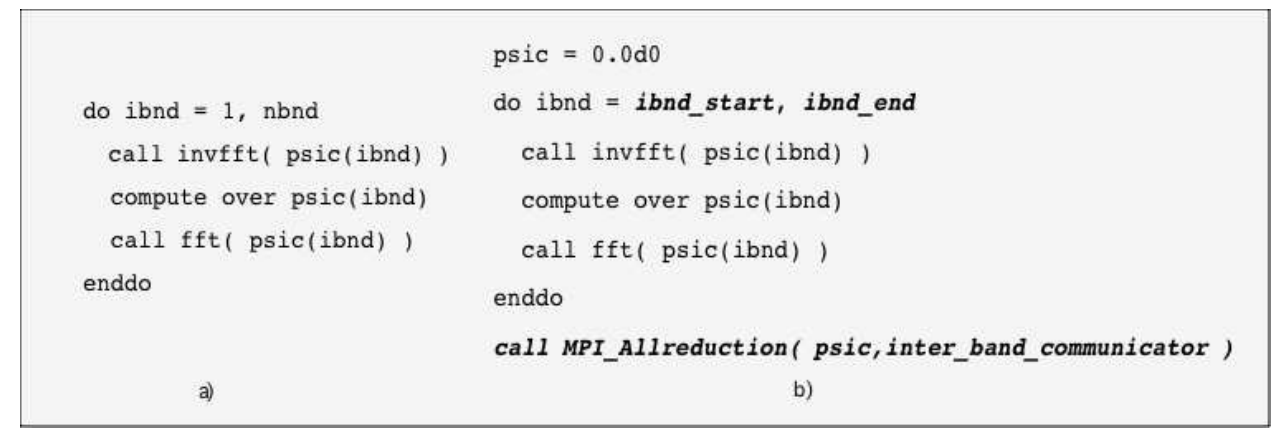

Figure 2: Code sections from QE-GIPAW code: (a) original implementation schema; (b) new implementation schema. The original number of iteration nbnd are divided evenly among the band groups used. So each group computes ibnd_end-ibnd_start iteration. At the ene of the loop the results of each group are summed up.

runs over all occupied electronic states. Previous to the present work, the Sternheimer equations were solved band-by-band by the Conjugate Gradient method, with a clever re-grouping of not converged bands, in order to exploit level-3 BLAS operations. In the new implementation, we further distribute the occupied bands over CPUs, and the CG algorithm has been modified to work with groups of bands. The new schema is reported as pseudo-language shape in Fig. 2. As the numbers of electronic bands become relevant the same model might also improves scalability of plane-wave DFT codes for efficiently runnig at thousands of cores in parallel.

The code analysis brought out that the GIPAW calculation is based on a simultaneous run over a 7-fold loop at one of the outermost routines. The image communicator (see Fig. 1), already implemented in the modular structure of the package, is designed for such purpose but it was not yet used for the QE-GIPAW code. A new communicator (later called electronic bands communicator) has been introduced in order to take advantage of this level of parallelism (see Fig. 1). As presented in the next section even higher scalability is reachable whether this is exploited. Despite the simple model, the introduction of new levels of parallelism required a considerable re-factoring due to the complexity of the software structure.

A similar parallelization scheme has been implemented for the Exact-Exchange (EXX) kernel of the PWscf code. We worked at the bands level as described for the QE-GIPAW code because the target was to enhance the parallelization for simulating big systems . As presented in Fig. 3 once again it was 


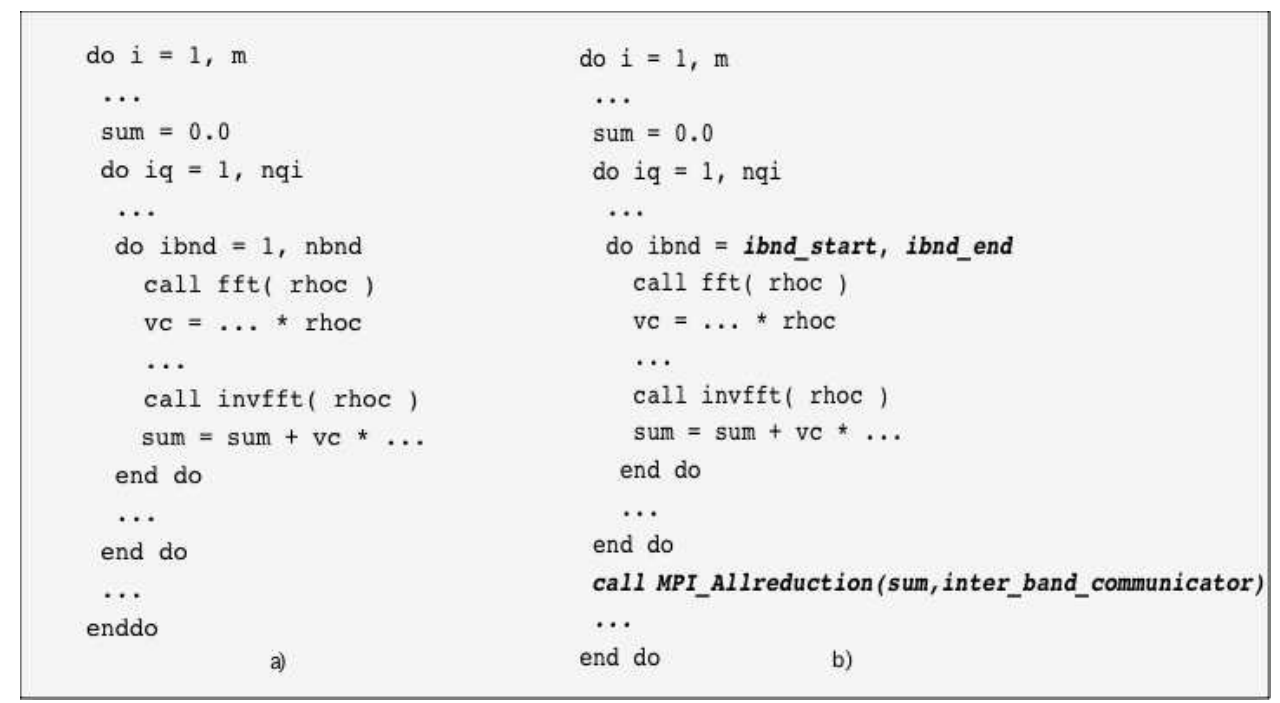

Figure 3: Code sections from EXX code: (a) original implementation schema; (b) new implementation schema. The original number of iteration nbnd are divided evenly among the band groups used. So each group computes ibnd_end-ibnd_start iteration. At the ene of the loop the results of each group are summed up.

possible to parallelize the inner loop over the electronic bands by introducing the electronic bands MPI communicator (i.e., by splitting the ibnd index across processors to perform on a different local section of the array rho). In this case the parallelization scales much more effectively because it is implemented on a high level loop that performs the whole computational workload of the EXX applications. In other words, the sum over occupied states that appear in the Green function calculation and in the EXX self-energy has been distributed across processors.

The CP code has benefited from a very similar parallelization strategy. At large scale, performance analysis underlined that main computational bottlenecks are: wave function orthogonalization (the use of ScaLAPACK could likely be for the better) and the evaluation of both forces on electronic degrees of freedom and charge density, specially for systems whith a very large number of electrons.

Both electronic forces and charge density are computed iteratively over the electronic bands, performing distributed 3D-FFT at each iteration. It is well-known that distributed 3D-FFT's do not scale because those are parallelized along the $\mathrm{z}$ axis and the number of points in this dimension does not grow at the same rate as the number of bands. As direct consequence, the overall scalability is limited by parallel FFT up to a few hundreds of tasks. A hybrid MPI+OpenMP parallelization scheme allows to extend the scalability up to few thousands of cores (see also CPMD [30, 31]). The band parallelization was introduced to scale the workload of the two loops which compute forces and charge density, likewise what we described for QE-GIPAW routines and of the EXX kernel, see Fig. 2 and Fig. 3. However, contrary to QE-GIPAW and the EXX kernel of PWscf, here, data and loops were distributed among the different band groups. Both data and parallelism over the g-vectors have been replicated, so that all the g-vectors loops are left untouched. Although the g-vectors are replicated across band group, the new implementation requires less memory. The wave functions arrays are distributed along two dimensions such g-vectors and band index. While two-dimensional arrays having number of atoms and band index as dimensions are distributed on the band index. Indeed, when the band parallelism is turned off the memory capacity is a limit for large physical system.

Beside the parallelization of electronic forces and charge density, the new schema has been partially used in the orthogonalization subroutine too. In particular band parallelization has been used to distribute the setup of the matrices for the orthogonalization (these matrixes are in fact the product of one wave function against all the other wave functions), whereas the diagonalization and the matrix multiplications performed with ScaLAPACK have been left replicated across the band groups.

In the next section we will show the scalability improvements obtained with these new parallelization schemes.

\section{Benchmark results}

Computer technologies are rapidly evolving and the number of compute cores needed to build the most powerful supercomputing infrastructure worldwide is drastically increasing (over a million for the time of writing). As a consequence, access to supercomputing petascale facilities commonly calls for specific requirements, that is scalability. Even for algorithms that are not embarassingly parallel, speedup can be achived for higher orders of magnitude of core count. However, in such cases, efficiency will be poor and so less useful as a measure. In such cases, the need of performing scientific challenges which would be impossible otherwise, it is a reasonable compromise to balance the cost of efficiency. Within this scenario, we enabled three scientific codes at petascale facilities so that the user community can finally exploit such compute capabilities. Validation and performance analysis of our development work was performed using real data. In particular, a cholesterol system with 592 atoms and 600 electronic bands was used for QE-GIPAW tests. The EXX tests have been performed on a 109-atoms a- $\mathrm{SiO}_{2}$ supercell with an oxygen interstitial, while 


\begin{tabular}{|l|l|l|l|l|}
\hline \# Cores & \# Threads & \# Bands & Time & Efficiency \% \\
\hline 64 & 1 & 1 & 621.15 & 100 \\
\hline 64 & 1 & 2 & 694.66 & 89 \\
\hline 128 & 1 & 1 & 533.33 & 58 \\
\hline 128 & 1 & 2 & 416.69 & 73 \\
\hline 256 & 1 & 4 & 300.79 & 57 \\
\hline
\end{tabular}

Table 1: GIPAW benchmark data for 592 cholesterol atoms on CURIE [9]. Wall-clock times are given in minutes.

\begin{tabular}{|l|l|l|l|l|}
\hline \# Cores & \# Threads & Time & Efficiency \% & Time Eq.10 \\
\hline 128 & 1 & 416.69 & 100 & 242.11 \\
\hline 896 & 1 & 81.59 & 73 & 58.71 \\
\hline 1792 & 2 & 51.82 & 58 & 37.60 \\
\hline 3584 & 2 & 36.00 & 41 & 24.81 \\
\hline
\end{tabular}

Table 2: GIPAW benchmark data for 592 cholesterol atoms on JUGENE [10]. Wall-clock times are given in minutes. Benchmarks were executed with a number of bands equal 2

for $\mathrm{CP}$, we have obtained a series of benchmarks results on a CNT10POR8 system (one hydrogen-saturated carbon nanotube, with four porphirin rings chemically linked to the CNT surface. The overall system comprise 1532 atoms, 5232 electrons, i.e, 2616 occupied bands). Calculations have been run on EU Tier-0 systems: CURIE [9], a 3-fraction linux cluster power by BULL and equipped with Intel x86-64 based technology , JUGENE [10] an IBM Blue Gene/P architecture and FERMI IBM Blue Gene/Q.

Tab. 1 shows the numbers measured performing GIPAW calculation on the CURIE Tier- 0 system. The first row presents the reference value obtained running over only two nodes. Indeed, 64 cores is the lowest value that would allow to perform such calculation within the 24-hours of wall clock limit and it is necessary to reach convergence to obtain timing information. The second row reports the elapsed time we got with the new version using two band groups, 32 cores for each group. Here the old approach still performs better since the diagonalization routine is not parallelized over the bands. However, by doubling the number of cores the parallelization over the electronic bands reduced the wall-time. The fourth row of Tab.11presents in fact better lower value of execution time and consequently a better efficiency ( 5 th column). De facto, 128 cores was the limit of the older version of the code as already at this stage efficiency is low. We obtain the same value of efficiency running the new version of the code at 256 cores using 4 bands group. The new level of parallelism introduced allows at large number of cores to increase the scalability by a factor of two. While the Green's function scales almost perfectly the code sections the iterative diagonalization is now the actual bottleneck. At this level $1 / 3$ of the overall time is spent to solve the diagonalization problem that does not take advantage from the parallelization over electronic bands. The parallelization over electronic bands cannot then be further applied to improve the scalability. So, QE-GIPAW runs efficiently with 2 band groups for this ex-

\begin{tabular}{|l|l|l|l|}
\hline \# Cores & \# Bands & Elapsed Time(s) & Efficiency \% \\
\hline 32768 & 64 & 523.45 & 100 \\
\hline 65536 & 128 & 311.59 & 84 \\
\hline
\end{tabular}

Table 3: EXX benchmark data for $109 \mathrm{SiO}_{2}$ atoms on JUGENE [10].

ample. This is the limit where users are suppose to utilize the other level of parallelism introduced.

As described in the previous section the code implements seven independent calculations at the outermost routine. For our experiments we took the limit at 128 cores. As shown in Tab.2, by using this new parallelism and running over 896 cores the code scales with $73 \%$ of efficiency respect to 128 cores (93\% if compared with the same number of cores while using the old version of the code). Although this is the best result reached in term of efficiency, further scaling with the introduction of hybrid MPI+OpenMP approach shows that is possible to reduce up to 36 minutes while running at 3584 cores. Thanks to the new development we present in this paper users can now easily scale at least 14 times the number of cores in regards to the old version of the same code.

The result analysis continues now with the EXX calculation. As described in the previous section the routine Vexx is carrying out almost all of the computational workload. In order to test the impact of the new parallelization we simulate a system of 108 atoms with 800 bands on the Tier-0 JUGENE machine. Despite the low number of atoms, full convergence requires a huge amount of core hours as shown in Tab. 3. almost 300,000 hours were necessary to complete this computation. For completeness it must be underlined that the current implementation allows only norm-conserving pseudopotentials [28] and by consequence the energy cutoff used for the fock operator (EXX operator) is the density cutoff, i.e. four times the wave function cutoff, although a smaller cutoff can be used.

The routine Vexx, that calculates the Exact-Exchange potential, scales perfectly by doubling the number of cores and the number of band groups. We measure an efficiency of around $\sim 97 \%$ moving from 32768 to 65536 cores if we compare the time of execution of the single Vexx routine. However, as presented in Tab. 3 also the global time scales almost perfectly at large number of cores. Indeed, since Vexx is extremely computationally intensive in regards to the overall time of execution, the code scales efficiently up to 65536 cores. The level of parallelism introduced by the new elctronic bands communicator definitely impacts more the EXX code than the QE-GIPAW code as in this case almost all of the computational workload can be parallelized over the electronics bands.

The benchmark analysis for the CP code, see Fig. 4, shows that, the time spent in the ortho subroutines decreases when increasing the number of cores, but not linearly, whereas the computation of dforce and rhoofr as well as other subroutine containing loops over electronic bands scale almost linerly with the number of cores. It is worth to note that, when increasing the number of band groups to the limit in which electronic force and charge density computation become negligible, the linear 


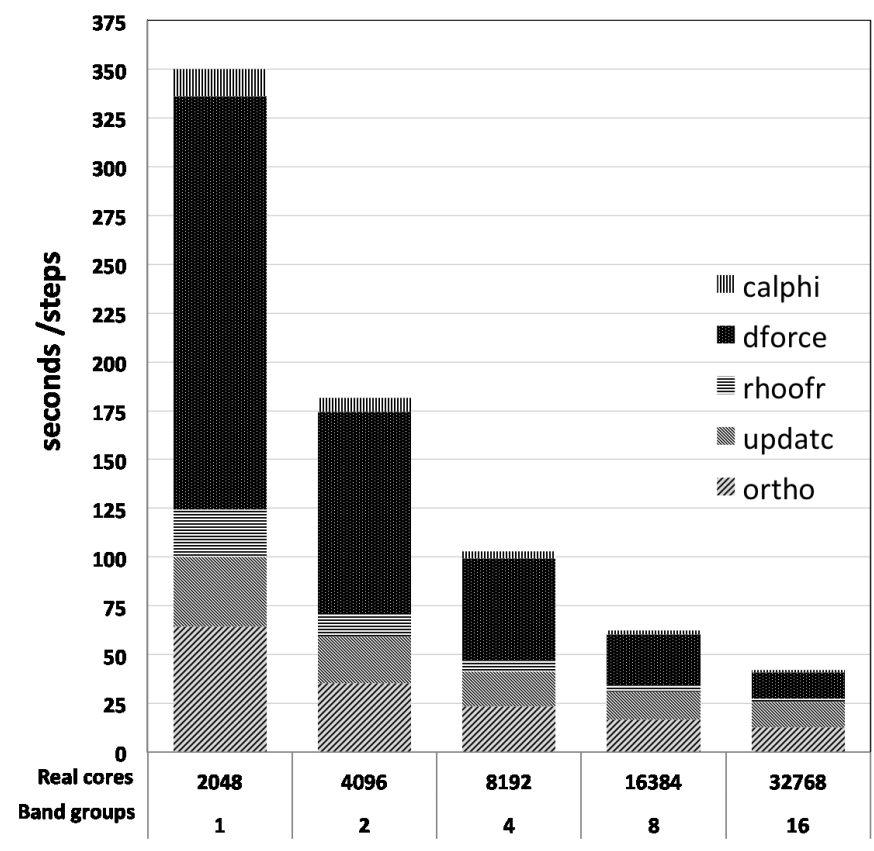

Figure 4: Scalability of the CP kernel of Quantum ESPRESSO on BGQ, using the benchmark CNT10POR8. A 1532 atoms system of an hydrogen-saturared chiral carbon nanotube, with four phorphyrin rings chemically linked to the surface of the nanotube

algebra computation contained in the ortho subroutine will become again the main bottleneck of the code. At that point some new strategy has to be implemented.

\section{Conclusions}

The work presented in this paper shows the advantage of the band parallelization approach to simulate challenging systems when the number of bands become huge. This was implemented for two DFT applications. (In fact, in order to accurately reproduce experimental data it is often necessary to keep the system size big enough). The results presented here suggest that it is mandatory to use this strategy on petascale hardware and beyond. Recently the memory distribution in some critical parts of Quantum-Espresso has been introduced. So, those two strategies coupled together are the workhorse that allow efficient simulation of DFT calculation on world-class supercomputers.

\section{Acknowledgements}

This work was financially supported both by the PRACE FirstImplementation Project funded in part by the EUs 7th Framework Programme (FP7/2007-2013) under grant agreement no. RI-261557 and by Science Foundation Ireland (grant 08/HEC/I1450). Benchmarks were carried out on the Jugene [10] machine at Jülich, on Curie [9] at the CEA and on Fermi[11]. DC is in indebted with Ari P. Seitsonen, Uwe Gerstmann and Francesco Mauri for coauthoring the first version of the QE-GIPAW code. We acknoweledge Emine Küçükbenli for sharing the cholesterol model, Arrigo Calzolari for the CNT10POR8 model, and Paolo Giannozzi for useful discussions. We acknowledge the Quantum ESPRESSO Foundation for the support.

\section{References}

[1] P. Giannozzi, S. Baroni, N. Bonini, M. Calandra, R. Car, C. Cavazzoni, D. Ceresoli, G. L. Chiarotti, M. Cococcioni, I. Dabo, A. Dal Corso, S. de Gironcoli, S. Fabris, G. Fratesi, R. Gebauer, U. Gertsmann, C. Gougoussis, A. Kokalj, M. Lazzeri, L. Martin-Samos, N. Marzari, F. Mauri, R. Mazzarello, S. Paolini, A. Pasquarello, L. Paulatto, C. Sbraccia, S. Scandolo, G. Sclauzero, A. P. Seitsonen, A. Smogunov, P. Umari and R. M. Wentzcovitch, J. Phys.: Condens. Matter 21, 395502 (2009); http://www.quantum-espresso.org

[2] P. Hohenberg and W. Kohn, Phys. Rev. 136, B864 (1964).

[3] W. Kohn and L. J. Sham, Phys. Rev. 140, A1133 (1965).

[4] X. Gonze, et al, Comp. Mat. Sci. 25, 478 (2002); also see http://www.abinit.org

[5] J.M. Soler, E. Artacho, J.D. Gale, A. García, J. Junquera, P. Ordejón, and D. Sánchez-Portal, J. Phys. Cond. Matter 14, 2745 (2002).

[6] R. Car and M. Parrinello, Phys. Rev. Lett. 55, 2471 (1985).

[7] G. Kresse and J. Hafner, Phys. Rev. B 47, RC558 (1993); G. Kresse and J. Furthmüler, 54, 1169 (1996).

[8] F. Gygi, IBM J. Res. Dev. 52, 137 (2008).

[9] http://www-hpc.cea.fr/en/complexe/tgcc-curie.htm

[10] http://www2.fz-juelich.de/jsc/jugene

[11] Fermi BG/Q, http://www.cineca.it/en/hardware/ibm-bgq

[12] F. Bottin, S. Leroux, A. Knyazev and G. Zérah, Comput. Mat. Sci., 42, 329 (2008).

[13] http://www.netlib.org/scalapack/

[14] P. J. Hasnip, M. I. J. Probert, K. Refson, M. Plummer anf M. Ashworth, Cray Users Group 2009 Proceedings (2009).

[15] E. J. Bylaskai, K. Tsemekhman, S. B. Baden, J. H. Weare and H. Jonsson, J. Comput. Chem. 32, 54 (2011).

[16] P. E. Blöchl, Phys. Rev. B 50, 17953 (1994).

[17] F. Mauri, B. G. Pfrommer, and S. G. Louie, Phys. Rev. Lett. 77, 5300 (1996).

[18] C. J. Pickard and F. Mauri, Phys. Rev. B 63, 245101 (2001).

[19] C. J. Pickard and F. Mauri, Phys. Rev. Lett. 91, 196401 (2003).

[20] J. R. Yates, C. J. Pickard, and F. Mauri, Phys. Rev. B 76, 024401 (2007).

[21] D. Ceresoli, A. P. Seitsonen, U. Gertsmann, F. Mauri, E. Kü cückbenli, S. de Gironcoli and N. Varini, http://qe-forge.org/projects/qe-gipaw

[22] D. Vanderbilt, Phys. Rev. B 41, 7892 (1990).

[23] A. D. Becke, J. Chem. Phys. 98, 1372 (1993).

[24] J. P. Perdew, K. Burke, and M. Ernzerhof, Phys. Rev. Lett. 78, 1396 (1997).

[25] S. Chawla and G. A. Voth, J. Chem. Phys. 108, 4697 (1998).

[26] M. C. Gibson, S. Brand and S. J. Clark, Phys. Rev. B 73, 125120 (2010).

[27] J. Gerrat and I. M. Mills, J. Chem. Phys. 49, 1719 (1968)

[28] N. Troullier, J. L. Martins, Phys. Rev. B 43, 1993 (1991).

[29] P. Giannozzi, F. De Angelis and R. Car, J. Chem. Phys. 120, 5903 (2004).

[30] J. Hütter and A. Curioni, Chem. Phys. Chem. 6, 1788 (2005);

[31] J. Hütter and A. Curioni, Parallel Computing 31, 1 (2005). 